\title{
LA ALIANZA CARDAMINION EN SIERRA NEVADA (ESPAÑA)
}

\author{
J. VARO y J.A. GIL (*)
}

\section{RESUMEN}

Se estudian las comunidades helofíticas, higro-esciófilas y terrisaxícolas de comportamiento microclimático, del piso supramediterráneo de Sierra Nevada. Asimismo se describen dos nuevas comunidades: la asociación Bryo-Plagiomnietum undulatae, propia de estaciones sometidas a un flujo continuo de agua en forma de goteo, y la asociación Pellio-Plagiomnielum rostratae de menores necesidades hídricas y propia de taludes cercanos a cascadas.

\section{RESUME}

On étude les groupements hélophitiques, hygro-sciaphiles et terrisaxicoles à comportement microclimatique, de l'étage supraméditerranéen de la Sierra Nevada. De même on decrit deux nouveaux groupements: l'association Bryo-Plagiomniclum undulatae, qui est reliée aux biotopes toujours humides par un dégouttement continue, et l'association Pellio-Plagiomnietum rostratae reliée aux pentes prochains des cascatelles, laquelle est moins hygrophile.

\section{INTRODUCCION}

La alianza Cardaminion Maas 1959 incluye asociaciones higro-esciófilas y meso-eutrofas, pertenecientes al orden Montio-Cardaminetalia (Paulowski 1928) Maas 1959 de la clase Montio-Cardaminetea Br. Bl. \& Tüxen 1943.

En Sierra Nevada, la alianza está representada por la subalianza BrachythecioCardaminenion Maas 1959, normalmente desarrollada sobre sustrato neutro o ligeramente ácido $(\mathrm{pH}=6,5-7,5)$. Asimismo reúne comunidades terri-saxícolas de composición brio-cormofítica, aunque en nuestro caso es mínima la incidencia de los taxones cormofíticos, como consecuencia de la falta de luz y escasez de suelo.

La alianza halla su óptimo sobre los protosuelos esquistosos del piso supramediterráneo de Sierra Nevada (1.000-2.000 m), estando en contacto con aguas mesoeutrofas que discurren por barrancos profundos y umbrios.

Como pertenecientes a la alianza Cardaminenion hemos estudiado las siguientes asociaciones: 


\section{Bryo pseudotriquetri - Plagiomnietum undulatae as. nova}

Holotypus: inventario n. ${ }^{\circ}$, tabla 1.

Sinecología: La asociación alcanza un óptimo desarrollo en taludes umbrios algo humificados y sometidos a un flujo de agua continuo, generalmente en forma de intenso goteo, siempre un tanto alejados del impacto directo de la corriente. Por estos motivos, la comunidad aparece colonizando profundas trincheras que suelen situarse bajo cascadas o en sus inmediaciones.

Sinfisionomía: Comunidad cerrada (cobertura media $=85 \%$ ), para una superficie de inventarios media de $11,1 \mathrm{dm}^{2}$, con un número específico medio de 5,4 (variaciones de 3 a 10). Se trata por tanto de una comunidad rica en especies y en la que dominan las Bryochamaephyta reptantia. El crecimiento estolonifero de Plagiomnium undulatum confiere a la asociación una fisionomía peculiar, estando sus tallos laxamente dispuestos en un primer estrato cuyo grosor puede alcanzar 5 o 6 $\mathrm{cm}$ y que generalmente cubre a un segundo estrato constituido por las Bryochamaephyta replantia: Brachythecium rivulare, Chiloscyphus polyanthus var. rivularis y Pellia epiphylia, así como la Bryochamaephyta caespitosa, Bryum pseudotriquetrum.

Sobre el tapiz briofítico destacan a veces Cardamine sylvatica y Stellaria alsine, pero sin que su presenci. llegue a influir decisivamente en la fisionomía general de la asociación.

Sindinámica: Por su crecimiento estolonífero, el hábitat de la asociación está colonizado en su mayor parte por Plagiomnium undulatum (V), bajo cuya protección se establecen Bryum pseudotriquetrum (IV) y Brachythecium rivulare (IV). Las tres especies mencionadas constituyen el núcleo principal de colonización de la asociación, las cuales favorecen la entrada posterior de Chiloscyphus polyanthus var. rivularis (III) y Pellia epiphylla (III). Asimismo la presencia de todas ellas permite la fijación gradual de un protosuelo, sobre el que se establecen otras especies de menores apetencias higrófilas, tales como Plagiomnium rostratum(I), Gymnostomum aeruginosum (I), Fissidens taxifolius var. pallidicaulis (I), Oxyrrbynchium praelongum (I) y Rhizomnium pseudopunctatum (I), las cuales tienen un comportamiento habitualmente mesófito y terrícola, que en latitudes de clima más húmedo colonizan los taludes de $\mathrm{pH}$ ácido; la xericidad ambiental de Sierra Nevada condiciona la presencia de aquéllas en microclimas húmedos, previamente colonizados por la asociación Bryo-Plagiomnietum undulatae.

Con cierta frecuencia, en estaciones donde el aporte hídrico es menor, se forma un complejo entre las asociaciones Bryo-Plagiomnietum undulatae y Pellio-Plagiomnietum rostratae. Por el contrario, en lugares más expuestos a la corriente, la formación de protosuelos es problemática y son preponderantes las especies reófilas de la clase Platyhypnidio-Fontinaletea.

Sintaxonomía: Las características ecológicas que definen a la asociación, así como el buen desarrollo logrado por especies como Brachythecium rivulare, Bryum pseudotriquetrum, Chiloscyphus polyanthus var. rivularis y Pellia epiphylla, creemos que sitúan a la comunidad en el ámbito de asociaciones pertenecientes a la suba- 
TABLA 1

\title{
BR YO-PLAGIOMNIETUM UNDULATAE as. nova
}

\author{
Número de inventario \\ Superficie $\left(\mathrm{dm}^{2}\right)$ \\ Cobertura (\%) \\ Exposición \\ Inclinación (으) \\ Sustrato \\ Número de especies
}

$\begin{array}{rrrrrrrrrrrrl}1 & 2 & 3 & 4 & 5 & 6 & 7 & 8 & 9 & 10 & 11 & 12 & \\ 36 & 25 & 25 & 30 & 20 & 16 & 30 & 20 & 15 & 10 & 15 & 20 & \\ 80 & 100 & 100 & 70 & 100 & 90 & 90 & 60 & 100 & 90 & 90 & 60 & \\ \text { NO } & \mathrm{S} & \mathrm{SO} & \mathrm{NO} & \mathrm{SO} & \mathrm{S} & \mathrm{SE} & \mathrm{NO} & \mathrm{S} & \mathrm{SE} & \mathrm{S} & \mathrm{S} & \\ 75 & 90 & 90 & 80 & 90 & 90 & 90 & 80 & 90 & 90 & 70 & 45 & \\ \mathrm{M} & \mathrm{M} & \mathrm{M} & \mathrm{M} & \mathrm{M} & \mathrm{M} & \mathrm{M} & \mathrm{M} & \mathrm{M} & \mathrm{M} & \mathrm{M} & \mathrm{M} & \\ 10 & 4 & 5 & 5 & 6 & 5 & 6 & 3 & 5 & 4 & 5 & 7 & 5,4\end{array}$

Caract. de asociación, subalianza y alianza: (Bryo-Plagiomnietum undulatae,

Brachythecio-Cardaminenion, Cardaminion).

Mnium undulatum

Bryum pseudotriquetrum

Brachythecium rivulare

Cardamine sylvatica

$$
\begin{array}{rrrrrrrrrrrrr}
1 & 2 & 3 & + & 3 & + & 3 & 2 & 3 & 2 & 2 & 3 & \mathrm{~V} \\
1 & \cdot & + & + & + & 2 & . & . & 2 & 2 & 2 & + & 1 V \\
1 & \cdot & 2 & 1 & + & 1 & 1 & . & . & 1 & 1 & 1 & 1 \\
1 & \cdot & . & . & 1 & \cdot & \cdot & 1 & . & . & + & . & 1 !
\end{array}
$$

Caract. de orden y clase: (Montio-Cardaminetalia, Montio-Cardaminetea)

Chiloscyphus polyanthus

$$
\text { var. rivularis }
$$

Pellia epiphylla

Stellaria alsine

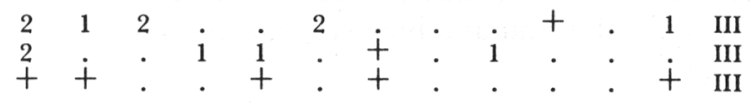

Caract. de Cratoneurion commutati:

Cratoneuron filicinum

2

Compañeras:

Platyhypnidium riparioides Mnium rostratum

Gy mnostomum aeruginosum

Fissidens taxifolius var. palidicaulis

Oxyrrhynchium praelongum

Mnium pseudopunctatum

Leyenda: $\mathrm{M}=$ Micasquistos.

Origen de los inventarios: Núms. 1, 4, 7: 1.600, 1.600, 1.300 m - Río Poqueira; 25/7\&75. Núms. 2, 9: 1.200 m - Río San Juan: 22/1/76. Núm. 3: 1.750 m - Río Chico de Soportújar: 6/7/75. Núms. 5, 6: $1.650 \mathrm{~m}$ - Río Lanjarón: 25/7/75. Núm. 8: $1.090 \mathrm{~m}$ - Río Genil: 16/1/76. Núms. 10, 11: 2.000 m - Río Válr: 8/6/76. Núm. 12: 1.000 m - Río Laroles: 4/3/76. 
lianıa Brachythecio-Cardaminenion de la alianza Cardaminion, orden Montio-Cardaminetalia, clase Montio-Cardaminetea.

Sincorología: La bibliografía consultada apenas proporciona datos precisos sobre la asociación. Sin embargo, la corología que establece MAAS (1959) para la alianza Cardaminion, puede servirnos de referencia a la hora de considerarla distribución de la asociación; en este sentido, es probable su presencia en el piso atlántico-colino de Irlanda, Bélgica, Francia y Holanda, en zonas montanas de Alemania, Checoslovaquia, Austria y Suiza, así como en los pisos subalpino y alpino de los Pirineos, Suiza, Austria, Checoslovaquia, Francia y Córeega.

\section{Pellio epiphyllae - Plagiomnietum rostratae as. nova}

Holotypus: inventario n. ${ }^{\circ}$, tabla 2.

Sinecología: Asociación asimismo terri-humícola y esciófila, aunque menos higrófila que la anterior, con la cual se halla en íntimo contacto. Suele establecerse en estaciones muy umbrias y húmedas, como son bordes de pequeños arroyos y taludes cercanos a cascadas, donde la influencia del agua se manifiesta esta vez en forma de salpicaduras o bien en pequeñas gotas suspendidas en el ambiente (aerohigrófila).

Sinfisionomía: Comunidad cerrada (cobertura media $=92,5 \%$ ), para una superficie de inventarios media de $26,2 \mathrm{dm}^{2}$, con un número específico medio de 7,8 (variaciones de 6 a 9). Como puede verse, se trata de una asociación algo más cerrada que la anterior, así como de una notable riqueza florística; este último aspecto puede relacionarse, con el conjunto de especies terrícolas y mesófitas que se añaden al núcleo principal de especies higrófilas que definen a la asociación.

En conjunto, en la asociación prevalecen los biótipos de Bryochamaephyta reptantia (50\%) que constituyen un tapiz cerrado; no obstante, las Bryochamaephyta caespitosa, Bryum pseudotriquetrum y Plagiomnium rostratum, se destacan de las anteriores en densos pulvinulos, desempeñando un papel fundamental en el desarrollo de la comunidad. La presencia de los hemicriptófitos Cardamine sylvatica y Montia rivularis, acentúan en cierto modo la heterogeneidad fisionómica de la asociación, pues aunque se presentan en forma aislada, no es por ello menos significativa la frecuencia con que lo hacen.

Sindinámica: La asociación inicia normalmente su desarrollo, mediante el establecimiento de cuatro especies pioneras que son las yuc inejor caracterizan la comunidad: Plagiomnium rostratum (V), Pellia epiphylla (V), Chiloscyphus polyanthus var. rivularis (IV) y Bryum pseudotriquetrum (IV). Todas se comportan como higroesciófilas y terrícolas, exceptuando Pellia epiphylla que en ocasiones actúa como terri-saxícola.

Generalmente, siempre que la asociación coloniza suelos de profundidad adecuada, es inmediatamente invadida por Oxyrrbynchium praelongum (V) y Fissidens 
TABLA 2

PELLIO-PLAGIOMNIETUM ROSTRATAE as. nova

Número de inventario

Superficie $\left(\mathrm{dm}^{2}\right)$

Cobertura (\%)

Exposición

Inclinación (으)

Sustrato

Número de especies

$\begin{array}{rrrrrrrrr}1 & 2 & 3 & 4 & 5 & 6 & 7 & 8 & \\ 20 & 20 & 30 & 40 & 40 & 20 & 20 & 20 & \\ 100 & 80 & 100 & 100 & 90 & 100 & 70 & 100 & \\ \mathrm{~S} & \mathrm{NE} & \mathrm{S} & \mathrm{SE} & \mathrm{S} & \mathrm{S} & \mathrm{SE} & \mathrm{NO} & \\ 90 & 90 & 90 & 45 & 90 & 80 & 90 & 90 & \\ \mathrm{M} & \mathrm{M} & \mathrm{M} & \mathrm{M} & \mathrm{M} & \mathrm{M} & \mathrm{M} & \mathrm{M} & \\ 7 & 9 & 6 & 8 & 7 & 7 & 6 & 6 & 7,8\end{array}$

Caract. de asociación, subalianza y alianza: (Pellio-Plagiomnietum rostratae, Brachythecio-Cardaminenion, Cardaminion).

Mnium rostratum

Pellia epiphylla

Bryum pseudotriquetrum

Brachythecium rivulare

Cardamine sylvatica

$\begin{array}{ccccccccc}1 & 1 & 3 & 3 & + & 2 & 2 & 3 & \mathrm{~V} \\ + & 1 & . & 2 & 2 & 3 & 1 & 1 & \text { V } \\ 1 & 2 & 3 & + & . & . & 2 & + & \text { IV } \\ + & 1 & + & 2 & . & . & . & . & \text { III } \\ . & + & . & + & 1 & + & . & . & \text { III }\end{array}$

Caract. de orden y clase: (Montio-cardaminetalia, Montio-Cardaminetea)

Chiloscyphus polyanthus var. rivularis

Montia rivularis

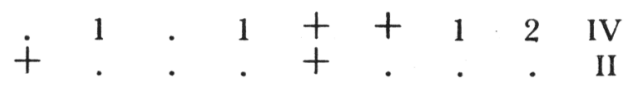

Compañeras:

Oxyrrhynchium praelongum $2+++21+\mathrm{V}$ Fissidens taxifolius var. palidicaulis

Platyhypnidium riparioides Mnium undulatum Nostoc sp.

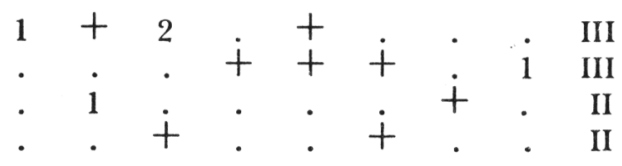

Leyenda: $\mathrm{M}=$ Micasquistos.

Origen de los inventarios: Núms. 1, 5, 6: $1.200 \mathrm{~m}$ - Río San Juan: 22/1/ 76. Núms. 2, 7, 8: $1.200 \mathrm{~m}$ - Río Alhorin: 16/7/76. Núm. 3: $1.800 \mathrm{~m}$ - Río Maitena: 13/7/77. Núm. 4: 1.750 m - Río Chico de Soportújar: 17/7/75. 
laxifolius (III), especies terrícolas y mesófitas que corresponden a las comunidades en contacto de Dicranelletalia. Las anteriores circunstancias favorecen en ocasiones el establecimiento de los hemicriptófitos Cardamine sylvatica(III) y Montia rivularis (II).

De otra parte, son frecuentes las estaciones donde la incidencia del agua es intermitente, en cuyo caso la asociación Pellio-Plagiomnietum rostratae se halla en ecotonía con la asociación Bryo-Plagiomnietum undulatae; ambas comunidades constituyen entonces un mosaico, en el que resulta difícil la individualización de las mismas. La situación anterior se puede complicar aún más con la presencia de Brachytbecium rivulare (III) y Platybypnidium riparioides (III), que encuentran acomodo en la asociación cuando ésta se localiza de forma más próxima al curso de agua.

Sintaxonomía: Las características ecológicas y composición florística de PellioPlagiomnietum rostratae están bastante relacionadas con las descritas para la asociación Bryo-Plagiomnietum undulatae; en consecuencia, ambas asociaciones tienen una posición sintaxonómica común: subalianza Brachythecio-Cardaminion de la alianza Cardaminion, orden Montio-Cardaminetalia, clase Montio-Cardaminetea.

Sincorología: Aunque difieren ecológicamente, las asociaciones Pellio-Plagiomnietum rostratae y Bryo-Plagiomnietum undulatae poseen una base florística semejante, por lo que creemos lógico atribuirles un areal común, es decir, en el piso colino de los países atlántico-europeos y montano de Centroeuropa; es asimismo probable su presencia en los tramos subalpino y alpino de los principales macizos montañosos europeos. 


\section{BIBLIOGRAFIA}

ALlORGE, P. -1947- Essai de Bryogéographie de la Péninsule Ibérique. P. Lectbezalicr. Paris.

BRAUN-BLANQUET, J. -1948- La végètation alpine des Pyrénées orientales. Monografías de la Eslación de Esludios Pircnaicos. Barcelona.

CASARES-GIL, A. -1914- Una excursión briológica a Sierra Nevada. Bol. R. Soc. Esp. Hisl. Nal., 14: 100-104. Madrid.

CASAS DE PUIG -1956- Aportaciones a la flora briológica de los Pirineos Centrales. Coll. Bot., 5: 419-424. Barcelona.

GEISSLER, P. -1976-Zur Vegetation alpiner Fliessgewasser. Mal. Flore Crypt. Suisse, 14 (2): 1-52. Teufen.

GIL, J.A. \& VARO, J. -1973- Contribución al estudio briológico de Sierra Nevada. II. El Barranco de San Juan. Trab. Dip. Bol. Unir. Granada, 2: 63-79. Granada.

HERTEL, E. -1974- Epilithische Moose und Moosgesellschaften in nordostlichen Bayern. Birl. Ber. Naturzwiss. Giss., 1. Bayréurh.

HUBSCHMANN, A.V. -1957-Zur Systematik der Wassermoosgesellschaften. Mill. flor. soz. Arl. gim., 6/7: 147-151. Stolzenau/Weser.

HUBSCHMANN, A.V. -1973- Moosgesellschaften des Nordwestdeutschen tieflandes zwischen Ems und Weser. Hirzogia, 3: 111-130

MAAS, F.M. -1954- Bronnen, Bronbeken, Bronbossen van Nederland, in het bijzonder die van de Veluwezoon. Een plantensociologische en oecolologische studie. Midid. Landbouwhogeschool Wageningen, 59 (12): 1-166.

PHILIPPI, G. -1965- Die Moosgesellschaften der Wutachschlucht.. Mill. bad. Landester. Naturkd. Natursch., 8: 625-668.

PHILIPPI, G. -1975- Quellflurgesellschaften der Allgauer Alpen. Bitr. Naturkd. Forsih. Sudw.-Dil., 34: 259-287.

QUEZEL, P. -1953-Contribution á l'étude phytosociologique et géobotanique de la Sierra Nevada. Mem. Soc. Brolcriana, 9: 5-78.

RIVAS MARTINEZ, S. -1975- Datos ecológicos sobre la vegetación acuática continental. Anal. Inst. Bol. A.J. Caruanilles, 23 (1): 199-205.

RIVAS MARTINEZ, S. -1981- Les étages bioclimatiques de la végétation de l'Espagne. Aitas III Congr. OPTIMA. Anal. Jard. Bol. Madrid, 37 (2). (En prensa). 